\title{
Hansen's Disease and Rheumatoid Arthritis Crossover of Clinical Symptoms: A Case Series of 18 Patients in the United States
}

\author{
Sarah M. Labuda, ${ }^{1 \star}$ John S. Schieffelin, ${ }^{1}$ Jeffrey G. Shaffer,${ }^{2}$ and Barbara M. Stryjewska ${ }^{3}$ \\ ${ }^{1}$ Tulane University School of Medicine, New Orleans, Louisiana; ${ }^{2}$ Tulane University School of Public Health and Tropical Medicine, \\ New Orleans, Louisiana; ${ }^{3}$ National Hansen's Disease Program, Baton Rouge, Louisiana
}

\begin{abstract}
Hansen's Disease (HD) is a rare, chronic granulomatous infection of the skin and peripheral nerves caused by the noncultivable organism Mycobacterium leprae. Arthritis is the third most common symptom of HD. Subjects with both confirmed HD on skin biopsy and chronic arthritis were identified at the National Hansen's Disease Program (NHDP). We conducted a series of medical chart reviews and extracted and logged personally deidentified data into a database and carried out descriptive analyses. Eighteen of 261 subjects presented to the NDHP with both HD and chronic arthritis between 2001 and 2015. Among these, 16 were male, 16 were white, and 15 were residents of Louisiana. The median age at diagnosis of HD was 67 years. Ten of these subjects were diagnosed with borderline lepromatous leprosy, seven were diagnosed with lepromatous, and one was diagnosed with borderline tuberculoid leprosy. Patients were symptomatic with arthritis for a median of 5.3 years before HD diagnosis. Sixty-two percent of patients (11) were diagnosed with rheumatoid arthritis (RA) before HD diagnosis, and 10 of which were seronegative RA. Hands, feet, wrists, and elbows were most commonly reported as affected joints. Over half of the patients $(61 \%)$ had completed HD multidrug therapy at the time of review, and $73 \%$ of these subjects had persistent joint pain requiring steroids or methotrexate for symptomatic control. Chronic arthritis in HD patients is present in a series of US-acquired cases of HD. Arthritis did not resolve with successful treatment of HD in most cases.
\end{abstract}

\section{BACKGROUND}

Hansen's Disease (HD) is a rare, chronic granulomatous infection predominantly of the skin and peripheral nerves caused by the noncultivable organism Mycobacterium leprae. Between 1994 and 2011, an incidence of 0.45 new cases per 1 million population was found in the United States (US). ${ }^{1}$ While HD is predominantly found in immigrants to the US from other more highly endemic countries, the infection is also endemic to the Gulf Coast States. ${ }^{1}$ Armadillos are the only nonhuman reservoir known to harbor the infection, and transmission is thought to be via respiratory droplets both between humans and in armadillo-to-human transmission. ${ }^{2}$ The incubation period of the disease is extremely long, often 2-10 years after exposure, and only about $5 \%$ of the population seems to be immunologically susceptible to developing symptomatic infection through poorly understood mechanisms. ${ }^{3}$ Most commonly, infected patients present with persistent macular rash with anesthetic patches due to preference of the organism for distal peripheral nerves in cooler regions of the body. ${ }^{3}$

Diagnosis is made through skin biopsy of the rash using Fite stain or PCR, and findings of organisms on Fite-stained specimens within cutaneous nerves on skin biopsy are pathognomonic for $M$. leprae. ${ }^{3}$ Treatment involves multidrug therapy (MDT) most often with dapsone, rifampin, and clofazimine - a drug with known anti-inflammatory effects-for 1-2 years to combat the infectious process, and may also require immunomodulators such as steroids, thalidomide, and methotrexate in the event of development of immunologic phenomena known as reversal reactions or erythema nodosum leprosum. ${ }^{3,4}$ This interaction of noninfectious inflammatory processes along with the direct damage done to the body by the organism itself can complicate both the diagnosis and treatment of HD.

*Address correspondence to Sarah M. Labuda, Tulane University School of Medicine, 1440 Canal Street, Suite 1600, New Orleans, LA 70112-2632. E-mail: slabuda@tulane.edu
After cutaneous and peripheral nerve manifestations, arthritis has been identified as the third most common symptom of $\mathrm{HD}$, leading to misdiagnosis as various rheumatologic disorders. ${ }^{5-9}$ Although acute arthritis may be seen with reversal reactions, chronic arthritis is also well-described in cases of HD. Chronic arthritis without destructive bony lesions on X-ray has been described as both an initial presenting sign as well as a later development in the process of HD. Distal joints are more frequently involved in published cases. ${ }^{6-9}$ Patients are occasionally found to produce antinuclear antibodies (ANA) and rheumatoid factor (RF), which are usually associated with autoimmune diseases such as rheumatoid arthritis (RA). ${ }^{5,6}$ It is uncertain if these antibodies are produced as a result of inflammation from the infection with $\mathrm{HD}$, or are indicators of underlying autoimmunity in the patient. Anticyclic citrullinated peptide antibodies (anti-CCP) are currently thought to be the most specific antibody for diagnosing RA and are reported to be negative in most cases of arthritis due to HD. ${ }^{6-9}$

There have also been rare cases of patients undergoing therapy for RA with various disease modifying antirheumatic drugs such as tumor necrosis factor alpha inhibitors which then unmasked or exacerbated previously unrecognized HD. ${ }^{9-15}$ A similar effect is seen with Mycobacterium tuberculosis, a much more common and better-known infection related to $M$. leprae. ${ }^{12}$ The interaction and overlap in symptoms of RA and HD is important for medical practitioners to recognize in various specialties of practice. Because a combination of treatment of the inflammatory arthritis and the underlying infection are necessary to resolve the symptoms, $\mathrm{HD}$ is an important, if infrequent, item on the differential diagnostic list of patients with rash and joint pain.

Single case reports of arthritis resembling rheumatic disease in HD patients have been published in the US, ${ }^{10,16}$ but larger series have been reported only in more highly endemic countries like Brazil and India. ${ }^{17}$ To our knowledge, this is the first report of a series of US-born HD patients with associated 
chronic arthritis. Through the resources of the clinical care physicians at the National Hansen's Disease Program (NHDP) in Baton Rouge, Louisiana, 18 adult patients with both biopsyconfirmed HD and polyarticular chronic arthritis, many meeting diagnostic criteria for RA, are described here.

\section{METHODS}

A retrospective chart review of patients with both chronic arthritis attributed to a rheumatologic diagnosis and confirmed HD seen at the NHDP, a national referral center for patients with HD providing clinical and ancillary care, from 2001 to 2015 was performed. A comparison to the U.S. Department of Health and Human Services report "A Summary of Hansen 's Disease in the United States-2004-2013" of 1,777 patients reported with HD across the US during that time period was also undertaken to determine if the chronic arthritis patients were similar to the general population of US HD patients. Approval through the Tulane University Institutional Review Board was obtained. Subjects with both confirmed HD on skin biopsy and chronic arthritis were selected based on tracking by the clinicians of the NHDP. Personally deidentified information was extracted from charts on standardized forms and logged into a database. Data were presented as medians and ranges or frequencies and percentages and basic descriptive statistics were performed. Fisher's exact tests were used to compare the HD cohort with chronic arthritis subgroup.

\section{RESULTS}

We identified 18 patients from the 261 seen from 2001 to 2015 at the NHDP in Baton Rouge, Louisiana, with both HD and chronic arthritis (Table 1 and Supplemental Table 1). Among these subjects, 16 (89\%) were male, 16 (89\%) were white, and $15(83 \%)$ resided in Louisiana both at birth and at the time of their HD diagnosis. The median age at diagnosis of HD was 65.5 years (range $49-86$ years). We compared data for our 18 subjects to comparable measures for the general US HD population. ${ }^{18}$ Among all cases diagnosed in the US, $23 \%$ were born in the US, and $8 \%$ were residents of Louisiana whereas the chronic arthritis cohort was $100 \%$ US-born and
$83 \%$ Louisiana residents $(P<0.001)$. All patients with both arthritis and HD were $>45$ years of age at diagnosis whereas only $46 \%$ of all cases in the US were above the age of 45 years at the time of diagnosis of HD $(P<0.001)$. Borderline lepromatous leprosy (BL) (Supplemental Tables 1 and 2) was diagnosed in $56 \%$ of chronic arthritis patients, but only $12 \%$ of all US HD cases are BL $(P<0.001)$.

Patients in this series had a median age of onset of joint pain of 62.5 years (range 47-78) and rash at the age 65 years (range 44-85). There was a median of 4 years' (range $0.4-13$ ) delay between the onset of symptoms and diagnosis with HD (Table 2). Dermatologists were by far the most common medical specialist $(88 \%)$ directly caring for HD patients with chronic arthritis at the time of diagnosis, although one patient was diagnosed on excision of a skin cancer lesion by a plastic surgeon, and one was diagnosed by a skin biopsy performed by their primary care provider. Eleven of $18(61 \%)$ patients were started on the first-line MDT regimen of clofazamine, rifampin, and dapsone for an expected 2-year course, and almost half (five of $11,45 \%$ ) required switching at least one of the drugs during the course of therapy because of intolerance (Supplemental Table 3). Six of 18 (33\%) patients were on alternative regimens, and one patient $(6 \%)$ was on two-drug therapy with rifampin and dapsone as indicated for borderline tuberculoid HD with a single skin lesion.

All but one patient (94\%) had an official diagnosis of at least one type of reversal reaction (Table 3; Supplemental Tables 3 and 4) in their chart, and all but one required at least steroids, methotrexate, or thalidomide to control reversal reaction and/ or arthritis symptoms. The majority of patients were prescribed two or more drugs during their course of MDT. The one patient who was not prescribed any medications by the physicians at the NHDP was documented to have been prescribed frequent courses of steroids for chronic obstructive pulmonary disease (COPD) exacerbations, which could also relieve their joint pain and swelling. Eleven patients (65\%) were prescribed methotrexate and steroids to control the reaction symptoms, followed by three (18\%) which required methotrexate, steroids, and thalidomide.

Two-thirds of patients (12 of 18) were followed by a rheumatologist at the time of diagnosis (Table 4). All 18 patients

TABLE 1

Characteristics of study subjects and general Hansen's disease (HD) cases reported in the United States (US) between 2004 and $2013^{23}$

\begin{tabular}{|c|c|c|c|}
\hline Characteristic & HD cases with arthritis, 2001-2015 $(N=18)$ & All reported HD cases, US, 2004-2013 ( $N=1,777)$ & $P$ value \\
\hline Male & $16(89)$ & $1,227(69)$ & 0.076 \\
\hline \multicolumn{4}{|l|}{ Ethnicity } \\
\hline Caucasian & $16(89)$ & $452(25)$ & $<0.001$ \\
\hline African American & $2(11)$ & $137(8)$ & - \\
\hline Other & $0(0)$ & $1,188(67)$ & - \\
\hline Above 45 years of age & $18(100)$ & $817(46)$ & $<0.001$ \\
\hline \multicolumn{4}{|l|}{ Place of residence } \\
\hline Louisiana & $15(83)$ & $138(8)$ & $<0.001$ \\
\hline Mississippi & $2(11)$ & $18(1)$ & - \\
\hline Florida & $1(6)$ & $171(10)$ & - \\
\hline Other US state & $0(0)$ & 1,049 (59) & - \\
\hline Other countries & $0(0)$ & $401(22)$ & - \\
\hline \multicolumn{4}{|l|}{ HD leprosy type } \\
\hline Lepromatous & $7(39)$ & $652(37)$ & 0.812 \\
\hline Borderline lepromatous & $10(56)$ & $222(12)$ & $<0.001$ \\
\hline Borderline tuberculoid & $1(6)$ & 232 (13) & 0.497 \\
\hline
\end{tabular}


TABLE 2

Diagnosis and treatment information on 18 patients with chronic arthritis and Hansen's disease (HD)

\begin{tabular}{lc}
\hline \multicolumn{1}{c}{ Characteristic } & \multicolumn{1}{c}{ Response } \\
\hline Onset of symptoms, median(range) & \\
Age at joint pain (years) & $62.5(47-78)$ \\
Age at rash (years) & $65(44-85)$ \\
Length of symptoms before diagnosis & $5.3(0.4-13)$ \\
$\quad$ with HD (years) & \\
Specialty diagnosing HD, N (\%) & $16(88 \%)$ \\
Dermatology & $1(6 \%)$ \\
Plastic surgery & $1(6 \%)$ \\
Family medicine & $11(61 \%)$ \\
Multidrug therapy (MDT) regimens, N (\%) & $5(45 \%)$ \\
Clofazimine, rifampin, and dapsone & $6(33 \%)$ \\
MDT changed because of intolerance & $1(6 \%)$ \\
Alternative regimens from initiation & 1 Tuberculoid leprosy on two-drug regimen \\
Tuberculoim & \\
\hline${ }^{\star}$ Reasons for intolerance of medications included anemia on dapsone and skin discoloration \\
due to clofazimine.
\end{tabular}

had documented joint pains, arthritis, and/or joint swelling along with skin lesions, although only two (11\%) reported fevers at any point, and seven (39\%) had numbness to skin lesions, extremities, or both (Supplemental Table 5). Sixtytwo percent of patients (11 of 18) were diagnosed with RA before diagnosis with HD, and among those, 10 of the 11 (91\%) were negative for RF. RF-positive RA, polymyalgia rheumatica, and psoriatic arthritis were diagnosed in one patient each, and five of the 18 patients did not have a rheumatologic diagnosis documented in the NHDP chart. Only two patients reported extra-articular manifestations of rheumatoid nodules, distinct from skin lesions of ENL, documented by the NHDP physicians. All 18 patients reported pain and swelling in more than one joint group, most commonly the small, more distal joints. Hands were reported to have pain and swelling in $14(78 \%)$ patients, followed by feet in $11(61 \%)$ patients. Wrists and elbows were reported to have pain and swelling in nine (50\%) and six (33\%) patients, respectively, and ankles and knees were least common at five $(28 \%)$ and one (6\%) patients each. Disease outcomes for most patients indicated persistent joint pains for those who

TABLE 3

Adverse reaction types and treatment medications among $N=18$ Hansen's disease cases with arthritis

\begin{tabular}{lc}
\hline \multicolumn{1}{c}{ Reversal reaction type } & Number reporting reaction (\%) \\
\hline At least one type of reaction & $17(94)$ \\
Type I & $2(11)$ \\
Type II & $7(39)$ \\
Neuritis & $1(6)$ \\
Type I and II & $2(11)$ \\
Type I and neuritis & $3(17)$ \\
Type II and neuritis & $1(6)$ \\
Type I, II, and neuritis & $1(6)$ \\
No reaction experienced & $1(6)$ \\
Medication for treating reaction & $1(6)$ \\
Steroids alone & $1(6)$ \\
Methotrexate alone & $11(61)$ \\
Steroids and methotrexate* & $1(6)$ \\
Steroids and thalidomide & $3(17)$ \\
Steroids, methotrexate, and & $1(6)$ \\
thalidomide & \\
None† & \\
\hline *One study subject briefly changed from methotrexate to lefleunomide at the time of \\
diagnosis.
\end{tabular}

TABLE 4

Rheumatologic characteristics of patients with Hansen's disease and chronic arthritis

\begin{tabular}{|c|c|}
\hline Characteristic & $N(\%)$ \\
\hline Followed by rheumatologist & $12(67)$ \\
\hline \multicolumn{2}{|l|}{ Patients with preexisting rheumatologic diagnosis } \\
\hline Rheumatoid arthritis (RA) (all categories) & $11(62)$ \\
\hline RA (seronegative) ${ }^{*}$ & $10(91)$ \\
\hline Polymyalgia rheumatica & $1(6)$ \\
\hline Psoriatic arthritis & $1(6)$ \\
\hline None & $5(28)$ \\
\hline Multiple/undetermined & $4(22)$ \\
\hline Rheumatoid factor results reported in chart & $14(78)$ \\
\hline Of the 14 with results, those with $R F+^{\star}$ & $1(7)$ \\
\hline $\begin{array}{l}\text { anticyclic citrullinated peptide antibodies (anti-CCP) } \\
\text { performed }{ }^{\star}\end{array}$ & $4(29)$ \\
\hline Of those with results, those anti-CCP+${ }^{*}$ & $0(0)$ \\
\hline $\begin{array}{l}\text { Laboratory results unavailable but patient followed } \\
\text { by rheumatologist }\end{array}$ & $3(17)$ \\
\hline \multicolumn{2}{|l|}{ Clinically joints involved (all had multiple joints) } \\
\hline Hands & $14(78)$ \\
\hline Wrists & $9(50)$ \\
\hline Elbows & $6(33)$ \\
\hline Feet & $11(61)$ \\
\hline Ankles & $5(28)$ \\
\hline Knees & $1(6)$ \\
\hline \multicolumn{2}{|l|}{ RA sequelae } \\
\hline Rheumatoid nodules & $2(11)$ \\
\hline \multicolumn{2}{|l|}{ Outcome of therapy } \\
\hline Deceased $\dagger$ & $2(11)$ \\
\hline Completed multidrug therapy & $11(61)$ \\
\hline Off all medications ${ }^{\star}$ & $2(18)$ \\
\hline Joint pain persists requiring steroids and/or methotrexate* & $8(73)$ \\
\hline Unknown* & $1(9)$ \\
\hline
\end{tabular}

completed MDT, with 11 of $18(61 \%)$ documented to have successfully finished at least 2 years of MDT, and eight (73\%) of those still requiring steroids and/or methotrexate, and the supervision of a rheumatologist, for joint pain and swelling. One had no documentation after completion of MDT, and two of the 11 were tolerating being off of all medication per last chart report. Of all the 18 patients, two are deceased, but neither died as a complication of HD.

\section{DISCUSSION}

Arthritis is the third most common symptom of HD, and as this series shows, patients often suffer long delays and misdiagnosis with other rheumatologic diseases when they have chronic arthritis with HD. Multiple case reports and series of chronic arthritis and HD have been published previously predominantly from the high-burden countries of India and Brazil, ${ }^{17,19-26}$ although a few case reports are from the $\mathrm{US}^{16}$ or other western countries ${ }^{27}$ among patients who have immigrated from other areas known to be endemic for HD. To our knowledge, this is the first series of cases of HD patients with chronic arthritis described in the US. Our population was overall older, more likely to have the BL form of HD, and more likely to have a course complicated by reversal reaction than the overall case information published for US HD cases. Generally, arthritis is reported to improve with successful treatment of HD, which was not apparent in our cohort of patients. Although our patient group's average age was 67, a series from Brazil has shown musculoskeletal manifestations of HD including chronic arthritis in children and young adults 
as well. ${ }^{6}$ Reports of possible "uncovering" of HD with immunosuppressive RA therapy has also been suggested. ${ }^{9-15}$ With our reported mean length of joint pain before HD diagnosis of 5.3 years, it seems unlikely that the immunonsupression led in the immediate sense to progression of symptoms of HD. However, the reported average assumed incubation period of HD is $2-10$ years, so this may represent an accelerated presentation while immunosuppressed compared with the usual natural history of disease progression. Finally, production of autoantibodies such as RF and ANA has been seen with HD in addition to cases of RA, confusing the distinction of whether there are two separate processes or one occurring in these patients. ${ }^{12}$ Almost all documented cases and series report negative anti-CCP in HD patients, although a few case reports have been published documenting patients with positive ANA, $\mathrm{RF}$, and anti-CCP in the setting of HD. ${ }^{6,8,9}$ Because it is now known that chronic inflammation and advanced age can lead to patients producing nonspecific antibodies like ANA and RF, and because anti-CCP is acknowledged to be the most specific antibody for RA, it seems possible that most of these patients have chronic arthritis due to HD with the confounding nonspecific production of antibodies.

Strengths of this study include the resources of the NHDP, a facility that specializes in the care of complicated HD patients both from Louisiana and across the US. Although it is not a representative sample of all HD cases in the US, the clinic in Baton Rouge sees the highest number of patients in a single facility in the continental US.

Limitations of this study include the selection of cases based on provider's tracking of cases and the limited sample size. These limitations may have led to biases in presenting only the most severe cases, or favoring patients who already were under the care of rheumatologists. Although numbers of patients with chronic arthritis and HD in this series are small compared with the total number of cases of HD in the US, there appear to be some differences in the characteristics of this patient group described above, perhaps limiting the generalizability to other populations of HD patients. Because we did not have access to the individual data for the general US HD population, purely exclusive comparisons between our study sample and the general US population were not possible. However, we suspect that the impact of this limitation is minimal because of the small study sample $(N=18)$ relative to the large sample for the general US population $(N=1,777)$.

A location bias may be present as well, because most of our patients were Louisiana state residents, whereas only $8 \%$ of all cases of HD in the US were attributed to Louisiana residents. This is likely due to the fact that the NHDP is located in Louisiana and more local patients are referred there, although less likely could represent a higher rate of HD patients with RA in Louisiana. The different time scale of chronic arthritis cases (2001-2015) versus national HD data (2003-2014) and the lack of individual-level data on the national comparison group limits the ability to make specific comparisons. However, because of the extremely long incubation period of the disease, the time scale is likely not a large confounding factor in the current comparison. Finally, most of our patients in the chronic arthritis group are also counted in the total number of HD patients nation-wide and, although close, our comparison group is from a slightly different time period.

There are several potential directions for future research on this topic. In 2008, Mycobacterium lepromatosis was identified as another organism causing clinical $\mathrm{HD},{ }^{28}$ and found to be just as common, if not more so, than $M$. leprae in clinical HD cases in Mexico. ${ }^{29,30}$ It is also now being more commonly found to be the etiologic agent of diffuse lepromatous leprosy and in case reports, has been found in patients with codiagnosis of RA. Further delineating the epidemiology of $M$. lepromatosis and its potential as a causative agent of a more severe clinical spectrum of HD, including in patients with chronic arthritis, would be helpful to understanding this ancient and elusive disease. Also, increasing evidence has shown the anti-inflammatory effects of minocycline ${ }^{31}$; this could add to the use of clofazimine, which is known to have anti-inflammatory effects as well as antimycobacterial effects, and potentially improve the recommended regimen for patients with reversal reactions as well as arthritis. Finally, further investigating whether these patients have severe arthritis due to their disease process, or actually have a separate rheumatologic disease, as well as the pathophysiology of the association of these two processes would be of great help to the practitioners treating these patients.

Although HD is rare, it is still endemic in some areas of the US and may have a spectrum of symptoms overlapping with rheumatologic diseases, especially seronegative RA. Dermatologists may often make the diagnosis, but HD should be in the differential of patients with rash and joint pain seen by generalists, emergency medicine physicians, and rheumatologists.

Received March 13, 2017. Accepted for publication August 4, 2017.

Published online October 23, 2017.

Note: Supplemental figures and tables appear at www.ajtmh.org.

Acknowledgments: We thank Russell Van Dyke for his assistance during the development of this manuscript.

Financial support: J. S. S. received financial support from NIH grant 5K12HD043451.

Authors' addresses: Sarah M. Labuda and John S. Schieffelin, Tulane University School of Medicine, New Orleans, LA, E-mails: slabuda@ tulane.edu and jschieff@tulane.edu. Jeffrey G. Shaffer, Department of Global Biostatistics and Data Science, New Orleans, LA, E-mail: jshaffer@tulane.edu. Barbara M. Stryjewska, National Hansen's Disease Program, Baton Rouge, LA, E-mail: bstryjewska@hrsa.gov.

\section{REFERENCES}

1. Nolen L et al., 2014. Incidence of Hansen's disease-United States, 1994-2011. MMWR Morb Mortal Wkly Rep 63: 969-972.

2. Truman RW et al., 2011. Probable zoonotic leprosy in the southern United States. N Engl J Med 364: 1626-1633.

3. Ridley DS, Jopling WH, 1966. Classification of leprosy according to immunity. A five-group system. Int J LeprOtherMycobact Dis 34: 255-273.

4. World Health Organization, 1998. WHO Model Prescribing Information: Drugs Used in Leprosy. Geneva, Switzerland: WHO.

5. Aletaha D et al., 2010. 2010 rheumatoid arthritis classification criteria: an American college of rheumatology/European league against rheumatism collaborative initiative. Arthritis Rheum 62: 2569-2581.

6. Ribeiro SL, Pereira HL, Silva NP, Neves RM, Sato El. 2008. Anticyclic citrullinated peptide antibodies and rheumatoid factor in leprosy patients with articular involvement. Braz J Med Biol Res 41: 1005-1010.

7. Zavala-Cerna MG et al., 2012. Anti-cyclic citrullinated peptide antibodies and rheumatoid factor sera titers in leprosy patients from Mexico. Rheumatol Int 32: 3531-3536. 
8. Guedes-Barbosa LS, Mangueira C, Scheinberg M, 2008. Anticitrulline peptide antibodies (CCP3) in leprosy sera: a negative association. Clin Rheumatol 27: 515-516.

9. Henriques CC, Lopez B, Mestre T, Grima B, Panarra A, Riso N, 2012. Leprosy and rheumatoid arthritis: consequence or association? BMJ Case Reports published online 13 August 2012.

10. Scollard DM, Joyce MP, Gillis TP, 2006. Development of leprosy and type 1 leprosy reactions after treatment with infliximab: a report of 2 cases. Clin Infect Dis 43: e19-e22.

11. Sobanko JF, Freeman AF, Palmore TN, Mendoza D, Richard Lee CC, Cowen EW, 2009. A Sri Lankan woman with rheumatoid arthritis and anesthetic plaques. J Am Acad Dermatol 60: 1018-1021.

12. Lluch $P$ et al., 2012. Development of leprosy in a patient with rheumatoid arthritis during treatment with etanercept: a case report. Semin Arthritis Rheum 42: 127-130.

13. Freitas DS, Machado N, Andrigueti FV, Reis Neto ET, Pinheiro MM, 2010. Hanseníase virchowiana associada ao uso de inibidor do fator de necrose tumoral: relato de caso. Rev Bras Reumatol 50: 333-339.

14. Lydakis $\mathrm{C}$ et al., 2012. Development of lepromatous leprosy following etanercept treatment for arthritis. Clin Rheumatol 31: 395-398.

15. Antônio JR, Soubhia RMC, Paschoal VDA, Amarante CF, Travolo ARF, 2013. Biological agents: investigation into leprosy and other infectious diseases before indication. An Bras Dermatol 88 (Suppl 1): 23-25.

16. Yens DA, Asters DJ, Teitel A, 2003. Subcutaneous nodules and joint deformity in leprosy: case report and review. J Clin Rheumatol 9: 181-186.

17. Salvi S, Chopra A, 2013. Leprosy in a rheumatology setting: a challenging mimic to expose. Clin Rheumatol 32: 1557-1563.

18. U.S. Department of Health and Human, 2011. A Summary of Hansen's Disease in the United States-2004-2013. 1-31.

19. Pereira HLA, Ribeiro SLE, Sato SE. 2008. Manifestaões Reumáticas da Hanseníane. ACTA Reum Port 33: 407-414.
20. Rath D, Bhargava S, Kundu BK, 2014. Leprosy mimicking common rheumatologic entities: a trial for the clinician in the era of biologics. Case Rep Rheumatol 2014: 1-5.

21. de Oliveira Fernandes RTM, Korinfskin JP, Espíndola MMM, de Oliveira Corrêa LM, 2014. Arthritis and diagnosis of leprosy: a case report and review of the literature. An Bras Dermatol 89: 323-325.

22. Sheetal S, Arvind C, 2009. Lest we forget Hansen's disease (leprosy): an unusual presentation with an acute onset of inflammatory polyarthritis and the rheumatology experience. Int $J$ Rheum Dis 12: 64-69.

23. Simeoni $S$ et al., 2011. Leprosy initially misdiagnosed as sarcoidosis, adult-onset still disease, or autoinflammatory disease. JCR J Clin Rheumatol 17: 432-435.

24. Miladi MI, Feki I, Bahloul Z, Jlidi R, Mhiri C, 2006. Chronic inflammatory joint disease revealing borderline leprosy. Joint Bone Spine 73: 314-317.

25. Prasad S et al., 2013. Leprosy revealed in a rheumatology clinic: a case series. Int J Rheum Dis 16: 129-133.

26. Vengadakrishnan K, Saraswat PK, Mathur PC, 2004. A study of rheumatological manifestations of leprosy. Indian J Dermatol Venereol Leprol 70: 76-78.

27. Kaur MR, Grindulis K, Maheshwari M, Ellis CJ, Bhat J, Tan CY, 2007. Delayed diagnosis of leprosy due to presentation with a rheumatoid-like polyarthropathy. Clin Exp Dermatol 32: 784-785.

28. Han XY et al., 2008. A new Mycobacterium species causing diffuse lepromatous leprosy. Am J Clin Pathol 130: 856-864.

29. Han XY, Zhang J, Li L, 2015. Leprosy agents Mycobacterium lepromatosis and Mycobacterium leprae in Mexico: a clarification. J Clin Microbiol 53: 3387-3388.

30. Han XY, Sizer KC, Velarde-Felix JS, Frias-Castro LO, Vargas-Ocampo $\mathrm{F}, 2012$. The leprosy agents Mycobacterium lepromatosis and $\mathrm{My}$ cobacterium leprae in Mexico. Int J Dermatol 51: 952-959.

31. Garrido-Mesa N, Zarzuelo A, Gálvez J, 2013. Minocycline: far beyond an antibiotic. Br J Pharmacol 169: 337-352. 